WIENER SLAVISTISCHES JAHRBUCH, Band 53/2007, 191-211

(C) 2007 by Österreichische Akademie der Wissenschaften, Wien

SERGIO BONAZZA

\title{
Kopitar und Friedrich Schlegel in Wien: Wissenschaftsbeziehungen
}

\begin{abstract}
Avtor trdi, da dosedanje mnenje o duhovni bližini in tesnem sodelovanju med Kopitarjem in Fr. Schleglom, kakor tudi o Schleglovem vplivu na Kopitarja, ne odgovarja stvarnosti, kajti konkretna dejstva dokazujejo ravno obratno. In sicer, da sta bila Kopitar in Schlegel na diametralno nasprotnih pozicijah tako na znanstvenem, kakor tudi na kulturnopolitičnem, nacionalnem in celo na verskem področju. Kopitarjev znanstveni vpliv na Schlegla je dokumentiran v dveh primerih: enkrat gre za vprašanje pomena italijanskega učenjaka L. A. Muratorija, drugič o vprašanju sorodnosti slovanskih jezikov s sanskritom. Zadnji primer je privedel Schlegla do tega, da se je začel učiti slovenščine. Kopitarjevi odnosi s Schleglom so bili predvsem rezultat Schleglovih iniciativ; to je razvidno tudi iz korespondence. Kopitarjevo sodelovanje pri Schleglovi reviji „Deutsches Museum“ ni imelo samo znanstvenega, ampak tudi polemičnega značaja. Dva od štirih Kopitarjevih člankov, objavljenih v tej reviji - Über die unmusikalische Beschaffenheit der deutschen Sprache in Die deutsche Orthographie. Von einem Kosaken, an den Herausgeber -, sta odgovor na Schleglovo trditev v njegovih „Dunajskih predavanjih“, da slovanski jeziki že po svoji naravi niso primerni za poezijo. Kaže, da Schlegel Kopitarjeve ironije ni doumel. Kopitar je imel tudi po Schleglovi smrti z njim opravka, ker je Schleglova vdova Dorothea hotela prodati dunajski dvorni knjižnici nekaj moževih rokopisov. Kot dodatek sta tukaj objavljeni dve še neobjavljeni Schleglovi pismi Kopitarju.
\end{abstract}

Das uns überlieferte Bild der Beziehungen Kopitars zu Friedrich Schlegel vermittelt, dass sie in einem sehr guten Verhältnis zueinander standen. Der ostdeutsche Historiker Eduard Winter, zweifelsohne der beste Kenner der deutsch-slavischen Kulturverhältnisse in der Donaumonarchie, der in den 60er Jahren als Erster die Kopitar-Schlegel Beziehungen untersucht hat, behauptet, dass Kopitar und Schlegel einander „geistig sehr nahe“ standen. „Wie eng beide zusammenarbeiteten“, zeige Kopitars Mitarbeit an der von Schlegel herausgegebenen Zeitschrift „Deutsches Museum“ (Winter 1968: 68-69). Ein Jahrzehnt später geht Winter noch einen Schritt weiter, wenn er feststellt, dass Schlegel einen starken Einfluss auf Kopitar hatte (Winter 1978: 208). Diese Sichtweise des Verhältnisses Kopitar-Schlegel ist dann in der Kopitariana übernommen worden. Jože Pogačnik behauptet in seiner KopitarMonographie, dass die Schriften Friedrich Schlegels Kopitar neue Gesichtspunkte eröffneten (Pogačnik 1978: 74). Ähnliches kann man auch bei einem anderen promi- 
nenten Kopitar-Forscher nachlesen: Stanislaus Hafner (Hafner 1983: 203, 207; Hafner 1997: 178-179).

Ich habe mir vorgenommen, die bestehenden Quellen zur Kopitar-Schlegel Beziehung zu überprüfen und sie hinsichtlich der darin aufscheinenden Widersprüchlichkeiten anders auszuwerten. Dazu habe ich auch neue, noch ungedruckte Quellen herangezogen. Mein Ziel war es, die Beziehung dieser beiden bedeutenden Persönlichkeiten der europäischen Romantik vollständiger darzustellen. Als neue Quellen sind hier Kopitars Briefe an Franz Bernhard Bucholtz gemeint, die ich im Nordrhein-Westfälischen Staatsarchiv im Münster entdeckt habe, dann Bucholtz-Briefe an Kopitar und die noch unveröffentlichten Briefe Fr. Schlegels an Kopitar, die ich in der Österreichischen Nationalbibliothek in Wien aufgefunden habe. Bucholtz war Historiker, ein Schüler Schlegels, der ihn nach Wien holte, wo er Sekretär in der Hof- und Staatskanzlei wurde und später die Position des Chefredakteurs der Wiener „Jahrbücher der Literatur“ innehatte.

Daraus ergibt sich ein völlig anderes Bild der Kopitar-Schlegel Beziehung. Von einer geistigen Nähe zwischen den beiden, oder vom „Einfluss“ Schlegels auf Kopitar sind keine Spuren zu finden. Das Gegenteil ist wohl der Fall. Zwischen Kopitar und Schlegel gab es in allen Bereichen tiefgreifende Divergenzen, so im wissenschaftlichen, kulturpolitischen, nationalen und sogar religiösen Bereich. Ich möchte dies mit Beispielen belegen.

Zum religiösen Bereich: Noch bevor Schlegel nach Wien zog, war er 1808 in Deutschland von der lutheranischen zur katholischen Kirche übergetreten. In Wien schloss er sich dann dem Kreis der ultrakonservativen Katholiken um Klemens Maria Hofbauer und den päpstlichen Nuntius Antonio G. Severoli an, die beide radikal-konservative Positionen innerhalb der katholischen Kirche vertraten. Diesem Kreis schlossen sich in den folgenden Jahren weitere norddeutsche Intellektuelle, fast alle Konvertiten, an, wie Zacharias Werner, Adam Müller, der in der Slovenistik wohl bekannte Friedrich August von Klinkowström ${ }^{1}$, natürlich auch der schon erwähnte Bucholtz ${ }^{2}$ und andere. ${ }^{3}$ Sie bildeten eine einflussreiche und zahlreiche Grup-

${ }^{1}$ Friedrich August von Klinkowström (1773-1835) war Leiter des Instituts für Knaben katholischer Religion, das Anfang Oktober 1813 durch die Initiative von Hofbauer in Wien gegründet wurde. Während der zehn Jahre seines Bestehens hat das Institut insgesamt 210 Zöglinge, darunter 143 adeliger Abstammung, aufgenommen. Unter den Zöglingen befand sich auch Anastasius Grün (Anton Graf Auersperg), der fünf Jahre in der Anstalt blieb (1819-1824). In den Jahren 1822-1824 war in der Anstalt als Instruktor der spätere slovenische Dichter Francè Prešeren, damals Jura-Student in Wien, tätig.

2 Aus dem katholischen Münster in Westfalen stammend, war Franz Bernhard von Bucholtz (1790-1838) wie selbstverständlich katholisch. Er gehörte schon in seinen jungen Jahren den konservativen katholischen Kreisen Deutschlands an, und es war kein Zufall, dass es kein geringerer als der Dichter Graf Friedrich Stolberg war, zweifelsohne der berühmteste Konvertite Deutschlands in der Zeit der Romantik, der Bucholtz Fr. Schlegel empfohlen hatte. Als er 1813 nach Wien kam, war er erst 23 Jahre alt.

3 Einen Sonderfall stellt in diesem Zusammenhang der in Augsburg geborene Privatsekretär Metternichs Joseph Anton Pilat (1782-1865) dar. Er war zwar katholisch, aber dem 
pe der deutschen Postromantik: das deutsche Mittelalter wurde mythisiert und die mittelalterliche Religiosität, auch „,das altdeutsche Christentum“ genannt, als spezifisch deutsche Religiosität aufgewertet (Mittner 1964: 808). Dem liberal denkenden Kopitar, Vertreter eines kritischen Katholizismus, ${ }^{4}$ war dieser Kreis ein Dorn im Auge. Deshalb versuchte er auch die Aktivitäten dieses Kreises nach Möglichkeit einzuschränken. Als er 1829 Chefredakteur der Wiener „Jahrbücher der Literatur“ wurde, weigerte er sich einige Beiträge von Autoren zu veröffentlichen, die diesem Kreis angehörten. Dies widerfuhr sogar einer Rezension des Schlegel-Schützlings und früheren Chefredakteurs dieser Zeitschrift Bucholtz über das Werk von Karl Adolf Menzel: Neuere Geschichte der Deutschen von der Reformation bis zur Bundes-Acte. Dort wurde das geistige Leben gänzlich auf kirchliche Bewegungen und Gegenbewegungen zu diesen reduziert. Solches geschah auch mit einem Aufsatz des deutschen Philosophen Windischmann über Friedrich Schlegel. Windischmann galt zudem damals als der bedeutendste katholische Philosoph Deutschlands. Das führte zu einer äußerst scharfen Polemik zwischen Kopitar und Bucholtz. ${ }^{5}$ Der Wiener Slavist blieb hart. Kopitars Meinung zu Schlegels Religionsanschauungen wird in einer

Katholizismus entfremdet. Im Jahre 1805 heiratete er in Berlin die protestantische Elisa (Elisabeth) von Mengershausen, deren Schwester Louise die Frau Klinkowströms geworden ist; als Pilat 1814 aus einem dienstlichen Aufenthalt in Paris zurück nach Wien kam, waren seine Frau, sein Schwager Klinkowström und dessen Gattin zum Katholizismus übergetreten, und er selbst fand unter der Anleitung Hofbauers den Weg zur Religion zurück. Obwohl er dem Kreis der ultrakonservativen Katholiken sehr nahe stand und mit Hofbauer, Fr. Schlegel, Z. Werner, Adam Müller eng befreundet war, vertrat er in religiösen Fragen nicht die konservativen Positionen; Pilat unterhielt freundschaftliche Beziehungen sowohl mit dem aus Breslau stammenden, aber dem Protestantismus treu gebliebenen Friedrich Genz, der in Wien im Staatsdienst war (Genz 1868), als auch mit dem weltoffenen Kopitar. Die noch unveröffentlichten Briefe Pilats an Kopitar werden in der National- und Universitätsbibliothek in Laibach unter der Signatur Ms. 579 aufbewahrt.

4 Vgl. dazu Kopitar an Johann Severin Vater, Wien, 17. Oktober 1815: „Der engl[ischen] Bibelgesellschaft hab' ich [...] den Vorschlag gemacht, für sie hier eine serbische, eine albanische, eine bulgarische und eine kroatische Bibelübersetzung zu veranstalten [...]. Ich habe freilich mehr die Sprachkenntnis, als die Bekehrung etc. zum Zwecke, wiewohl ich übrigens ein grosser Verehrer des reinen Christenthums bin (was aber in der Bibel stark mit Spreu gemengt ist, weswegen ich zum Wohl und zur Ehre desselben eine neue stark expurgirte Bibel wünschte, was aber freilich wieder starke Haken hat, und als Bibel wohl unausführbar ist)" (Ziegengeist 1983: 526). Siehe auch Kopitar an J. Fesl, Rom, 20. Mai 1837: „Dem Fräulein $\mathrm{H}^{* *}$ können Sie sagen, dass ich am Pfingstsonntag die Messe Sr. Heiligkeit selbst in der Capella Sistina auf ihre Intention gehört habe; bezahlt habe ich sie freilich nicht, dafür fordere ich aber auch nichts, als höchstens ein memento vice versa. Aber es war kein Spaß, das Ding dauerte von 10 bis 1 Uhr; mit Einschluss einer monotonen, gebärdelosen Respectpredigt coram Pontifice, die mir ein halblautes aber deutsches hol dich der T[eufel] auspresste" (Hafner 1969: 31). Siehe dazu auch Bonazza 1980: 91-93.

5 Vgl. Kopitar an Bucholtz, Wien, 27. April 1829, undatierter Brief, 30. April 1829, in: Nordrhein-Westfälisches Staatsarchiv, Münster, Nachlass Bucholtz, 247 (Kopitar) und Bucholtz an Kopitar, Wien, 30. April 1829, 4. Juli 1829, in: Österreichische Nationalbibliothek, Wien, Authogr. 13/34-4,5. 
ironisch-spöttischen Bemerkung über Schlegel deutlich, die wir in einem Schreiben vom 24. Juni 1814 an Josef Dobrovský lesen können:

Ihr Jude Wolf hat nun hier die fantastischen Katholiken kennen gelernt, Schlegel, P. Hofbauer, Nuncius \&c., und - geht nach Berlin, um ihnen dort Anekdoten von uns zu erzählen. Die Gabe zu lügen hat er auch reichlich. Es werden schöne Sachen herauskommen. Schlegel als Beichtkind des olim Einsiedlers Hofbauer, mit dem Rosenkranz in der Hand, und dem Kreuzmacher auf bäuerische Art, als künftiger Stifter eines neuen Mönchsordens, oder doch Staatssekretär S. Heiligkeit etc. (Jagić 1885: 386-387)

Betrachten wir nun die Differenzen zwischen Kopitar und Schlegel auf dem Gebiet der Sprachwissenschaft: Im Jahre 1808, kurz bevor er nach Wien übersiedelte, hatte F. Schlegel in Heidelberg das Werk Über die Sprache und die Weisheit der Indier veröffentlicht. Dort behauptet er, dass das Sanskrit mit der lateinischen und griechischen, mit der germanischen und persischen Sprache die größte Verwandtschaft aufweist. Dagegen stellt er fest, dass mit der armenischen, den slavischen, keltischen und mit den semitischen Sprachen entweder sehr geringe Gemeinsamkeiten bestehen oder gar keine (Schlegel 1975b: 114). Diese unwissenschaftliche Behauptung Schlegels, der es noch dazu gewagt hatte, über slavische Sprachen Urteile abzugeben, ohne sie selbst zu kennen, konnte den temperamentvollen und nach wissenschaftlicher Exaktheit strebenden Wiener Slavisten nicht gleichgültig lassen. Kopitar polemisierte mit Schlegel, bekämpfte seine unwissenschaftliche Behauptungen in der Sanskrit-Frage und macht sich auch über ihn lustig, wenn er ihn als „unseren einzigen Inder“6 bezeichnet. Von seinen Auseinandersetzungen mit Schlegel über die Sanskrit-Frage informierte Kopitar auch den Berliner Altphilologen Friedrich Wolf in einem Brief aus dem Jahre 1811:

Wir Slaven behaupten gegen Fr. Schlegel, dem Sanskrit noch viel näher verwandt zu seyn, als Griechen und Germanen. (Vasmer 1939: 112)

Die auseinandergehenden unterschiedlichen Wertungen zwischen Kopitar und Schlegel reichen auch tief in nationale und kulturpolitische Fragen hinein. Wenn Kopitar vom „Vaterland“ spricht, meint er damit die gesamte Donaumonarchie mit ihren deutschen und nichtdeutschen Ländern. Wenn Schlegel vom „Vaterland“ spricht, versteht er darunter ausschließlich Deutschland und den deutschen Teil des österreichischen Kaiserreichs. Die Sehnsucht nach der verlorenen deutschen Einheit wie sie im Rahmen des Heiligen Römischen Reiches Deutscher Nation bestand, wird bei ihm in dieser Zeit stark spürbar. Im Jahre 1810 hielt Schlegel in Wien die Vorlesungen Über die neuere Geschichte; in der fünften Vorlesung, Karl dem Großen gewidmet, kommt dieser Gedanke deutlich zum Ausdruck:

Nun kam jene, in frühern Zeiten oft vergeblich gesuchte große National-Einheit aller deutschen Völker zu Stande. In diesem Zeitraum, von dem durch Wahl der ganzen Nation auf

${ }^{6}$ Vgl. Kopitar an Václav Hanka, Wien, 3. Juli 1819: “[...] quare exspectemus dum cum imperatore redierit Fridr. Schlegel, unicus noster Indus; nimis enim periculosum est divinando tentare rem arbitrariam, quin totum scribendi systema noris.“ (Jagić 1897: 32) 
den Thron erhobnen Könige Konrad, bis auf Kaiser Rudolph den Ersten, war Deutschland der mächtigste Staat in Europa, und kaum wird sich in der Geschichte ein andres Beispiel auffinden lassen, daß auf irgend einem Throne eine solche fast ununterbrochene Reihe kraftvoller Helden und großer Herrscher auf einander gefolgt wäre, als in diesem großen, freien, und während eines Zeitraums auch fest verbundenen Wahlreiche. (Schlegel 1846c: 102)

Dieser Gedanke hing eigentlich auch mit seinem Entschluss zusammen, das geopolitisch zersplitterte Deutschland zu verlassen und nach Wien zu übersiedeln, überzeugt, dass nur hier, unter dem Schirmschutz der kaiserlichen Krone des Heiligen Römischen Reiches Deutscher Nation und der römisch-katholischen Kirche, zukunftsträchtige Formen einer gesamtdeutschen Ordnung entwickelt werden können.

1812 gründete Schlegel in Wien die Zeitschrift „,Deutsches Museum“. Anders als üblich, werden in der Vorrede des Januar-Heftes des ersten Jahrgangs keine konkreten Hinweise über die Zielsetzung der Zeitschrift gegeben. Es wird lediglich erwähnt, dass Geschichte, Philosophie, Literatur und Kunst jene Gegenstände sind, denen diese Zeitschrift sich widmen wird (Schlegel 1975a: 220). Erst im NovemberHeft desselben Jahrgangs, genauer in der ,Ankündigung' des Zweiten Jahrgangs 1813 (S. 463), ist zum ersten Mal vom Ziel der Zeitschrift die Rede:

Der Zweck dieser Zeitschrift ist im Allgemeinen der: Deutsche Sprache und Geschichte, deutsche Kunst und Erkenntnis nach unsern besten Kräften zu befördern, und alles Vorzügliche, dahin Wirkende zur allgemeinen Kunde und Anerkennung zu bringen. Wir möchten für so vieles Einzelne Gute und Schöne, was in deutscher Art und Sprache gedacht und hervorgebracht worden, oder noch gedacht und hervorgebracht wird, einen gemeinschaftlichen Mittelpunkt aufstellen, die zerstreuten geistigen Kräfte des Vaterlandes immer mehr vereinigen, und eben dadurch auch den Geist und selbst die Gesinnung der Nation aufrecht erhalten und befestigen. (Schlegel 1812: 463)

Und zwei Monate später in der Vorrede des Januar-Heftes des zweiten Jahrgangs [1813] ergänzt Schlegel die Zielsetzung der Zeitschrift und präzisiert einige Details: mit der Gründung des „Deutschen Museums“ wäre

[...] zuerst der feste Grund gelegt [...], auf welchem das ganze Gebäude einer neuen deutschen Geistesbildung, einer bessern Erkenntnis und Gesinnung, einer wahrhaft deutschen Kunst und Rede sich erheben und emporwachsen kann. (Schlegel 1975a: 282)

In seinen Richtlinien stellt Schlegel auch eine unterschiedliche Literaturrezeption zwischen österreichischen und deutschen Lesern fest und meint:

Es wäre wohl überhaupt Zeit, dass dieser Unterschied in der Literatur allmählich ganz aufhörte, und wir in der Geistesbildung ungetrennt, mehr und mehr eine Nation wären und würden, wozu nach allen Kräften beizutragen der vornehmste Zweck des MUSEUMS ist. (Schlegel 1975a: 287)

Zur gleichen Zeit, in der Schlegel das „Deutsche Museum“ plante und herausgab, entwickelte Kopitar seine kulturpolitischen Vorstellungen des Austroslavismus, wonach Österreich und nicht Russland Zentrum des Slaventums werden sollte, denn in Österreich würden fast alle slavischen Sprachen gesprochen, in Russland dagegen nur eine. Außerdem wäre Österreich nicht nur geopolitisch, sondern auch historisch berechtigt, Mittelpunkt des Slaventums zu sein, weil das Altkirchenslavische, die 
erste Literatur- und Kultursprache der Slaven, auf österreichischem Gebiet (Pannonien) entstanden sei. ${ }^{7}$ Im Rahmen seines kulturpolitischen Programms des Austroslavismus plante Kopitar in Wien eine slavische Zentralakademie mit Zweigstellen in allen peripheren slavischen Zentren der Habsburgischen Monarchie.

Unmittelbar nach dem Erscheinen des Januar-Heftes des zweiten Jahrgangs des „Deutschen Museums“ berichtete Kopitar seinem Landsmann Jakob Zupan (Brief vom 17. Februar 1813) folgendermaßen davon:

Das deutsche Museum gibt Schlegel in Wien heraus. Wien ist nun das Centrum Deutschlands in so mancher Rücksicht. (Jagić 1897: 252)

Diese Worte zeigen klar, welche Meinung Kopitar über Schlegels Bestrebungen hatte, aus Wien den Mittelpunkt des Deutschtums zu machen. Die Erscheinung des „Deutschen Museums“, die Kopitar früher wohlwollend begrüßt hatte, empfand er jetzt als störend für seine kulturpolitischen Ideen. Sein oben zitierter Brief an Zupan beweist das deutlich.

Soviel über die tiefgreifenden Divergenzen zwischen Kopitar und Schlegel.

Schlegel und Kopitar kamen im selben Jahr, 1808, nach Wien. Schlegel war 36 Jahre alt, Kopitar 28. Beide hatten offen erklärte Ziele und geheime Wünsche. Schlegels offizieller Grund war es, in Wiener Archiven historische Forschungen zur Verfassung einer Monographie über Kaiser Karl V. durchzuführen. Sein unausgesprochener Wunsch war es aber, in Wien eine kulturpolitisch bedeutsame Position zu erreichen. Kopitar kam offiziell nach Wien, um in der Kaiserstadt Rechtswissenschaften zu studieren; sein Wunsch war es aber, Bibliothekar in einer großen Bibliothek zu werden, womöglich an der Wiener Hofbibliothek (Jagić 1885: 3-4). Beide haben relativ schnell ihr Ziel erreicht: Schlegel wurde 1809 in den österreichischen Staatsdienst mit dem Rang eines Hofsekretärs aufgenommen, Kopitar wurde 1810 zum vierten Skriptor der Hofbibliothek ernannt. Der Kontakt zwischen Kopitar und Schlegel kam ziemlich bald zustande im Einverständnis und zur Zufriedenheit beider. Der jüngere Kopitar war offensichtlich froh, und auch stolz, mit dem berühmten und hochgeschätzten deutschen Romantiker Umgang haben zu können. Er erwähnt Schlegel zum ersten Mal in einem Brief vom 14. April $1809^{8}$ an seinen Mäzen Baron Sigismund Zois, um ihm mitzuteilen, dass „Genz und Schlegel in Lager des Prinz Karl ${ }^{\text {“9 } 9}$ waren. Ein Jahr danach äußert Kopitar in einem weiteren Brief an Zois vom 16. Februar $1810^{10}$ seine Unzufriedenheit über die zögernde Art und Weise mit welcher Franz Sartori, der Herausgeber der „Annalen der Literatur und Kunst in den österreichischen Staaten" seine Rezensionen veröffentlicht und meint, er würde sie

\footnotetext{
${ }^{7}$ Zum Thema Kopitar und der Austroslavismus vgl. Winter 1958, Hafner 1963, Pogačnik 1982, Bonazza 1983, Bonazza 1988a.

8 Kidrič 1939: 195.

9 Mit dem „Lager des Prinz Karl“ war das Kriegslager Erzherzog Karls im Jahre 1809 in den Schlachten von Aspern und Wagram gemeint.

10 Kidrič 1941: 141.
} 
zurück verlangen und Schlegel geben, der in der Zwischenzeit eine neue Zeitung, den „Oesterreichischen Beobachter“", gegründet hatte. Das geschah zwar nicht und Kopitar veröffentlichte seine Beiträge nach wie vor in den „Annalen“ und in anderen Zeitschriften. Die Bemerkung Kopitars beweist jedoch, dass in dieser Zeit Schlegel für ihn eine logistische und kulturelle Stütze darstellte. Zwar hat Kopitar im „Oesterreichischen Beobachter" zur Zeit der Schlegelschen Redaktion im Jahre 1810 nichts veröffentlicht, er ist dann aber Mitarbeiter bei einer anderen, von Schlegel 1812 gegründeten Zeitschrift, eben dem oben erwähnten „Deutschen Museum“, geworden. Und gerade die Mitarbeit beim „Deutschen Museum“ stellt zweifelsohne das interessanteste und zugleich das merkwürdigste Kapitel der Kopitar-Schlegel Beziehung dar. Im Oktober-Heft des ersten Jahrgangs des „Deutschen Museums“ (S. 342-343), noch bevor die kulturpolitische Zielsetzung der Zeitschrift bekannt geworden war, veröffentlichte Kopitar seinen ersten Beitrag: Über ein österreichisches Idiotikon. An den Herausgeber. ${ }^{11}$ Die Verfassung eines österreichischen Idiotikons war ein altes wissenschaftliches Desiderat Kopitars. Der unmittelbare Anlass seines Aufsatzes war die Erscheinung eines Werkes von Franz Josef Stalder in demselben Jahr: Versuch eines schweizerischen Idiotikons, mit etymologischen Bemerkungen untermischt. Sammt einer Skizze einer schweizerischen Dialektologie. Kopitar bringt in dem Aufsatz sein Anliegen vor, eine geeignete Person möge ,etwa nach dem Muster des schweizerischen“ ein österreichisches Idiotikon verfassen. Aus diesem Grunde wendet er sich an Schlegel mit dem Wunsch, der Herausgeber des „Deutschen Museums“ möge ,,als geachteter Sprachkenner“ den Österreichern „,ans Herz legen“, ein solches Idiotikon ,abfassen“ zu wollen.

Schlegel veröffentlichte die Anmerkungen des Herausgebers unmittelbar in Anschluss an den Artikel Kopitars. Darin erklärt er mit viel Eifer, auf welche Weise ein österreichisches Idiotikon am besten entstehen könnte, und meint, dass die Gesamtredaktion des ganzen Unternehmens von einem einzigen Gelehrten geleitet werden müsste,

[...] wozu gewiß der gelehrte Herr Einsender [= Kopitar], wenn seine anderen Geschäfte es ihm erlauben, besonders geeignet wäre. (Schlegel 1975a: 275)

Schlegel äußert also allen Ernstes die Überzeugung, dass der Slavist Kopitar „,besonders geeignet wäre“ die Hauptredaktion eines österreichischen Idiotikons zu übernehmen. Es stellt sich verständlicherweise die Frage, warum das österreichische Idiotikon Kopitar am Herzen lag. Die Antwort auf diese Frage gibt uns er selber in der Besprechung des oben erwähnten schweizerischen Idiotikons von Stalder, die er für die „Wiener allgemeine Literaturzeitung" verfasste: Bei den innerösterreichischen Slaven gibt es Wörter und Wendungen, wie z. B. vasvati / dörfen (auf Besuch gehen oder Besuch bekommen), Türken / turšica (Mais), Tobak trinken / tobak piti,

${ }^{11}$ Nachgedruckt in: Miklosich 1857: 177-178. 
die bei den anderen Slaven unbekannt sind, deshalb wäre ein österreichisches Idiotikon nicht nur für Innerösterreicher interessant, sondern auch für die Slavisten. ${ }^{12}$

Zwischen Kopitar und Schlegel häuften sich mit der Zeit die unterschiedlichsten kontroversen Sichtweisen. Anfänglich waren es wissenschaftliche und literarische Ansichten, die Kopitar als Meinungsverschiedenheiten betrachtete; deshalb hatten diese auch keine Auswirkungen auf ihre Beziehung. Später kamen auch kulturpolitische und nationale Anschauungen dazu, die jetzt aber nicht ohne Folgen blieben. Der Wendepunkt war das November-Heft des ersten Jahrgangs [1812] des „,Deutschen Museums“, wo in der ,Ankündigung' des Zweiten Jahrgangs 1813 (S. 463) zum ersten Mal die programmatischen Richtlinien der Zeitschrift dargestellt wurden. Der Inhalt der ,Ankündigung', und noch deutlicher die zwei Monate danach im Januar-Heft des zweiten Jahrgangs [1813] erschienene Vorrede, zeigten Kopitar klar und eindeutig, dass die Divergenzen zwischen ihm und Schlegel keineswegs nur literarischer Natur waren, sondern dass grundsätzliche weltanschauliche Auffassungen und kulturpolitische Ansichten im Spiel waren. Jetzt erfahren bei Kopitar auch die früheren literarischen „Meinungsverschiedenheiten“ eine völlig neue Interpretation, besonders manche von Schlegel in seinen Wiener Vorlesungen aufgestellten Behauptungen. Von da an beginnt Kopitars innere Loslösung von Schlegel, ohne dass dieser selbst es merkte. Noch am 14. Oktober 1812 schrieb Kopitar seinem Landsmann Zupan nach Laibach:

Cum Schlegelio prandi nuper apud Humboldt legatum Prussiae. Ita me persequuntur hi Tedeschi. Schlegelio scripsi publice (in deutschem Museo) de idiotico austriaco, et ipse publice respondit cum multis complimentis, quibus tua vanitas patriotica, si legeret, mire gloriaretur (Jagić 1897: 247).

Es war das allerletzte Mal, dass Kopitar mit Stolz über seinen Umgang mit Schlegel spricht. Ab 1813 wird Schlegel in der Kopitar-Korrespondenz meistens mit ironischem Unterton erwähnt. Unabhängig davon war Kopitar, als Angestellter an der Wiener Hofbibliothek, sozusagen prädestiniert, mit dem prominenten Bibliotheksbenützer Kontakte zu haben. Trotz ihrer grundverschiedenen kulturpolitischen und wissenschaftlichen Auffassungen bestanden zwischen Kopitar und Schlegel intensive wissenschaftliche Kontakte und sogar dauerhafte Formen der Zusammenarbeit. ${ }^{13}$ Diese waren häufiger von Schlegel angeregt und gewollt. Gewöhnlich trafen sie sich in der Hofbibliothek.

${ }^{12}$ Kopitar, Deutsche Sprache. Versuch eines schweizerischen Idiotikons, mit etymologischen Bemerkungen untermischt. Sammt einer Skizze einer schweizerischen Dialektologie. Von Franz Josef Stalder, Dekan und Pfarrer zu Escholzmatt im Entlebach. I. Bd. 507. II. XII u. 582 S. Aarau, bei Heinrich Remigias Sauerlander. 1812. 8, in: „Wiener allgemeine Literaturzeitung“", 17. Mai 1814, Nr. 39, S. 617-624. Nachgedruckt in: Miklosich 1857: 265-272.

13 Davon berichten unter anderem auch Kopitars Briefe an Josef Dobrovský, Václav Hanka, Franc Metelko, Jakob Zupan, Jacop Grimm und Friedrich Wolf (Jagić 1885: 324, 343344, 373, 386-387; Jagić 1897: 32-33, 42, 173, 234, 247, 252; Vasmer 1938: 16; Vasmer 1939: 112). 
Die Zusammenarbeit zwischen Kopitar und Schlegel war wegen Schlegels aprioristischer Geringschätzung der Slaven einigermaßen erschwert. In den schon erwähnten Vorlesungen Über die neuere Geschichte, gehalten in Wien 1810, zeichnet Schlegel ein sehr negatives Bild von den Slaven, diesen „nicht edlen“ und „nicht freien Leuten“. ${ }^{14}$ Sozusagen ein Gegenbild zu dem sehr positiven, das Herder im „Slavenkapitel“ seines Werkes Ideen zur Philosophie der Geschichte der Menschheit (1784-1791) aufgestellt hatte. Wenn man dann noch dazu Schlegels abwertende Betrachtung des Volksliedes ${ }^{15}$ dazunimmt, wird klar, dass damit ein Thema ausgeklammert werden musste, das für Kopitar eine zentrale Bedeutung hatte: das serbische Volkslied.

Kopitar widmete einen beträchtlichen Teil seiner wissenschaftlichen Tätigkeit der serbischen Volksdichtung. Er war es, der auf entscheidendste Weise Vuk Karadžić zur Erstellung seiner Volksliedersammlung angeregt hatte und dann auch dieses Werk im deutschen Sprachraum verbreitet und bekannt gemacht hat. In seinem Werben um die Slavistik in Deutschland konnte er bis zum Erscheinen der altkirchenslavischen Grammatik von Josef Dobrovský im Jahre 1822 fast nur auf das serbische Volkslied zurückgreifen, wie er selbst zugibt:

14 „Man hat den Deutschen der damaligen Zeit einen Vorwurf aus der Unterdrückung und harten Behandlung der Slawen gemacht; in Rücksicht auf den Krieg selbst, der hartnäckig und mit Erbitterung geführt war, mag der Vorwurf gegründet sein. Für die Wirkung auf die Folgezeit darf man nur den blühenden Wohlstand so mancher ursprünglich slawischen Länder im nördlichen Deutschland, die durch deutsche Eroberer oder Colonisten, ganz oder halb deutsch geworden sind, mit andern Ländern vergleichen, welche die alte slawische Einrichtung durchaus beibehalten haben. Bei den slawischen Völkern war ursprünglich die Trennung der Adeligen und der Nichtadeligen weit strenger, das Verhältnis der ersten zu den letzten ungleich drückender. Daß wie in Deutschland, ein Stand der Freien bei den Slawen ursprünglich Statt gefunden habe, findet sich keine Spur. Von der innern Stärke, dem Wohlstande, und der damaligen Bevölkerung Deutschlands, muß die weite Ausbreitung deutscher Ansiedelungen in Ländern, die zuvor slawisch waren, wie Brandenburg, Meißen, Schlesien, einen großen Begriff geben. Bloße Eroberung ohne nachfolgende zahlreiche Ansiedelung hätte das nie bewirken können. Dazu rechne man noch die großen Heere, welche nach Italien und in den Kreuzzügen von Deutschland ausgingen. Auf die deutsche Verfassung hatte aber die Einverleibung der slawischen Länder und Völker unstreitig eine nachtheilige Wirkung. Von zwei Seiten drang jetzt ein Einfluß in Deutschland ein, welcher der alten Freiheit entgegen war. Von der einen Seite der neue fränkische Lebensgeist, vor welchem der Stand der Freien immer mehr verschwand; von den andern, ein nach slawischer Sitte härterer Druck der nicht edlen, nicht freien Leute“ (Schlegel 1846c: 121-122). Vgl. auch Winter 1978: 206.

15 „Die Volkslieder sind als einzelne poetische Anklänge einer der Poesie günstigern Vorzeit von großem Werth; doch ist es an sich immer nicht das rechte Verhältniß, wenn die Poesie, welche den Geist und das Gefühl der gesammten Nation ergreifen, rege erhalten, und weiter entwickeln soll, dem Volke allein überlassen bleibt. Auch werden solche einzelne verlorne poetische Anklänge, mit der Zeit immer mehr unverständlich; sie finden sich am häufigsten bei solchen Nationen, deren Sinn zwar poetisch ist, deren Poesie, Sage und ganze National-Erinnerung aber, etwa durch lange Bürgerkriege, oder durch eine allgemeine Erschütterung und Veränderung der Denkart, unterbrochen und zerstückelt worden ist." (Schlegel 1846a: 239) 
Mit dem Slavischen rathe ich Ihnen allen, solange noch zu warten, bis unser Abbé Dobrowský in Prag mit seiner von mir zugleich betriebenen Gramm(atik) des slaw. Bibeldialekts, seculi IX, fertig wird, was diesen Sommer geschieht. Dann werde ich alle meine deutschen Freunde von Breslau bis Oxford zum Slav(ischen) Studio auffordern, aber itzt wüßte ich ihnen nicht zu sagen, womit sie anfangen sollen.

Die Serbischen Lieder sind nach meinem Gefühle einzig; und wenn die Liebeslieder, wie jener Rec(ensent) sagt, nur mit dem hohen Liede sich vergleichen lassen, so kommen die Heldenlieder im Ausdruck und im Gedanken der Ilias am nächsten. (Ziegengeist 1988: 713)

Schlegel hat in seinen Vorlesungen über die Geschichte der alten und neuen Literatur, gehalten in Wien 1812, lediglich dem spanischen Volkslied und der nordischen (germanischen) Heldendichtung literarische Würde zuerkannt. Weil die spanischen Volkslieder

[...] nicht bloß Volkslieder sind in dem beschränkten Sinne des Wortes, sondern die besten derselben sind in einer größern und allgemeinern epischen Weise gedacht und abgefaßt, und wahrhaft national, dem Volke klar und anziehend, für die Gebildetsten aber im Sinn und Ausdruck edel genug [...] (Schlegel 1846a: 239),

und weil in der nordischen Heldendichtung, oder Ritterpoesie, das „Wunderbare“ einen „,ernsten Sinn“, die ,innere Wahrheit“" und die „Bedeutung“ hat (Schlegel 1846b: 28-29). Kopitar, der seinem Landsmann Jakob Zupan davon berichtete (Brief vom 19. Februar 1812), fügte hinzu, dass solche Heldenlieder wie die der Skandinavier, die Schlegel jetzt aufwertet, auch die Kroaten besäßen und ein ähnlicher Geist, der die isländischen Sagen durchdränge, auch im Igorlied zu finden sei (Jagić 1897: 234).

Für die serbische Volksepik, für welche sich sogar Goethe ${ }^{16}$, J. Grimm ${ }^{17}$ und viele andere deutsche Gelehrte und Literaten ${ }^{18}$ begeistern konnten, zeigte Schlegel kein Verständnis. Die slavische Welt blieb ihm nach wie vor völlig fremd. Denn es ist doch merkwürdig, dass nach zehnjährigem Aufenthalt in Wien, der Name Josef Dobrovský ihm noch kein Begriff war. Als 1823 Dobrovský ihm seine altkirchenslavische Grammatik für seinen Bruder August Wilhelm geschickt hatte und er den Bruder davon in Kenntnis setzte, schreibt er den Namen des berühmten tschechischen Philologen mit „Dombrowsky“ (Schlegel 1980: 412). Dobrovský war nicht nur in den wissenschaftlich gebildeten Kreisen Wiens bekannt, sondern auch bei der allgemeingebildeten Öffentlichkeit, denn über ihn haben ab und zu die Wiener Zeitungen berichtet. So berichtete z. B. die damals berühmteste Wiener Literaturzeitschrift „Annalen der Literatur und Kunst in dem Oesterreichischen Kaiserthume“ in ihrem April-Heft 1812 (S. 89), dass die russische Universität zu Char'kov den Abbé Dobrovský zu ihrem Ehrenmitglied gewählt hat. Diese Nachricht erschien in der Zeitung nur wenige Monate nachdem Fr. Schlegel in ebenden ,Annalen der Litera-

\footnotetext{
${ }^{6}$ Milović 1941a, Milović 1941b.

7 Schulte-Kemminghausen 1958, Mojašević 1990: 43-47, 64-88.

18 Ćurčin 1905: 146-223, Bonazza 1988b.
} 
tur und Kunst“ (Dezember-Heft 1811, S. 358-359) die Ankündigung seines „Deutschen Museums" erscheinen ließ.

Andere deutsche Gelehrte, die sich zur selben Zeit länger in Wien aufgehalten hatten, haben die für die Slavenkunde einmalige Quelle, die Kopitar und seine Tischrunde im Stammlokal „Zum weißen Wolf“ darstellten, ganz anders genutzt. So hat Wilhelm von Humboldt in den Gesprächen mit Kopitar wichtige wissenschaftliche Erkenntnisse gewonnen und neue Anregungen bekommen, die seine Sprachtheorie entscheidend beeinflusst haben (Mattson 1975: 306); Leopold Ranke hat in Wien zwei Werke verfasst - Don Carlos und Die Serbische Revolution -, die direkt auf die Kontakte mit Kopitar und seinem Kreis zurückzuführen sind (Bonazza 1990: 168-170); Jacob Grimm hat in Wien begonnen, sich mit dem Südslavischen zu beschäftigen (Bonazza 1990: 167-168); Hoffmann von Fallersleben, Autor des Gedichtes „Deutschland, Deutschland über alles“, erzählt in seinen Memoiren begeistert von einer neuen, faszinierenden Welt, die sich ihm durch die Teilnahme an der Tischrunde im „Zum weißen Wolfen“, wo sich die Elite der slavischen Intellektuellen der Kaiserstadt zu treffen pflegte, aufgetan hat (Hafner 1957: 188). Schlegel hat in den zwei Jahrzehnten, während er in Wien weilte, dieses kulturelle Angebot nicht wahrgenommen.

Schlegel war aber für andere, nichtslavistische Gegenstände, die im Interessensbereich Kopitars lagen, wie z. B. die Werke des italienischen Historikers, Gelehrten und Literaten Ludovico Antonio Muratori (1672-1750), des Begründers der wissenschaftlichen, sich auf Quellen stützenden Historiographie, aufnahmebereit. In einem Brief an Kopitar aus dem Jahre 1821 bittet Schlegel der ,Zusage gemäß, nun auch um 4ten Band des Muratori“" (Schlegel 1980: 349). Der deutsche Romantiker hat bis dahin Muratori weder in einem seiner Werke erwähnt, noch in einem seiner zahlreichen Briefe zitiert. Das ist auch verständlich, wenn man bedenkt, dass Muratori in den konservativen Kreisen Italiens als Ketzer und Atheist galt. Kopitar hingegen hielt Muratori für einen der bedeutendsten Autoren überhaupt. In seinem ersten Brief an Dobrovský vom 30. März 1808, noch bevor er von Laibach nach Wien übersiedelte, als er seinem „Meister“ den eigenen Lebensplan schilderte und den Wunsch äußerte, Bibliothekar in einer reichen Bibliothek zu werden, womöglich an der Wiener Hofbibliothek, ergänzt er den Brief mit der Aussage: „um der slavischen Geschichte das zu werden, was Muratori der italienischen ist“" (Jagić 1885: 4). In Kopitars Grammatik der slavischen Sprache in Krain, Kärnten und Steyermark, die ein Jahr später erschien, sind deutliche Spuren von Muratoris Ideen feststellbar; und zwar auf S. XVIII, wo er über die Wechselbeziehungen zwischen „Sprache“ und „Kultur“, bzw. zwischen Sprache und geistiger Entwicklung reflektiert. Wenn Kopitar schreibt:

[...] noch keine Nation der Welt ist der Barbarey durch Mathematik entrissen worden: die Natur verändert ihren Lauf nicht, und durch schöne Künste und Wissenschaften, durch schöne Schriftsteller und Dichter sind Griechen und Römer, Italiener, Franzosen, Engländer und Deutsche cultiviert worden! [...] 
kann man nicht umhin an Muratori zu denken, der in seinem Werk Della perfetta poesia italiana (Modena 1706) sagt:

$[\ldots]$ le lingue allora sono salite in alto pregio, quando elle hanno avuto più scrittori eccellenti, che con esse abbiano trattato tutte le scienze e arti. ${ }^{19}$

Kopitars Beschäftigung mit Muratori beschränkte sich nicht nur auf seine Bildungsjahre, sondern dauerte bis ins reifere Alter an. Das geht aus seiner Korrespondenz deutlich hervor. So erfährt man aus einem Brief Kopitars an den Präfekten der Bibliothek „Marciana“ in Venedig, Pietro Bettio vom 16. November 1821, dass der deutsche Historiker Georg Heinrich Pertz in italienischen Archiven Forschungen für die Sammlung Monumenta Germaniae historica durchführt und somit Muratori nachahmt (Bonazza 1980: 162). Weiter vermittelt uns Kopitars Brief an seinen Freund Josef Fesl vom 16. Juli 1837, dass er auf seiner Italienreise im Jahre 1837 sowohl in Rom als auch in Bologna ein Werk Muratoris intensiv suchte, ohne es jedoch gefunden zu haben (Hafner 1969: 40). Wie man sieht, Muratori war für Kopitar eine Inspirationsquelle und ein Vorbild. Das bestärkt die Deutung, dass Schlegels Interesse für Muratori höchstwahrscheinlich auf Kopitars Einfluss zurückzuführen ist.

Schlegel dürfte vorher von Muratori nicht viel mehr als den Namen gekannt haben. Das geht unter anderem auch aus seinen „Schriften aus dem Nachlass“" hervor. In den Fragmenten zur Geschichte und Politik. Erster Teil (Schlegel 1995a) auf S. 393 hat Schlegel unter der Bezeichnung „Historische und literarische Notizen aus Joh. v. Müllers Briefen“ folgende Aufzeichnung hingeschrieben:

S. 229. Kaiser Octavianus auch eine wilde Geburt einer Mittelalterseinbildung und in ursprünglicher Gestalt auf d. berühmten Bibliothek befindlich, welcher Muratori vorstund.

Auf S. 395 findet sich folgender Satz, den Schlegel aus einem Brief Johannes von Müllers an Bonstetten von 1798 abgeschrieben hat:

Hier (Wien) fing ich an, die Quellen für Österreich und Ungarn zu studieren; diese leiteten mich auf Byzanz; dieses große Corpus in das Reich der Araber, wobey Spezialschriftsteller über Persien, Palästina, Afrika und Arabisch-Spanien mitgenommen wurde. So kam ich zu meinem oft unterbrochenen Muratori zurück, und will diesen vorerst beenden.

Auch in den Fragmenten zur Geschichte und Politik. Zweiter Teil (Schlegel 1995b) wird Muratori kurz zitiert. Auf S. 168 findet sich unter der Bezeichnung „Aus Schlözers Weltgeschichte" folgende Aufzeichnung:

Oberlin Orbis Antiqui monumentis suis illustrati primae lineae. Argent. Eherne Schlange in Mayland. Murat. Antiqu. Ital. V. p. 73.

Die Aufzeichnungen sind in den Jahren 1812/13 und 1815 gemacht worden, und Muratori scheint nicht Hauptgegenstand der Anmerkung zu sein. Schlegel enthält sich auch irgendeines Kommentars darüber, und in seinen, im Druck erschienenen historischen Schriften (Schlegel 1966) wird Muratori nicht erwähnt.

19 Vgl. dazu Bonazza 1980: 17-20, 22-23. 
Die oben erwähnte Diskussion zwischen Kopitar und Schlegel über die Frage der Verwandschaft der slavischen Sprachen mit dem Sanskrit blieb für den deutschen Romantiker nicht ohne Folgen. Er beschloss eine slavische Sprache zu lernen und wählte das Slovenische, Kopitars Muttersprache. Es ist belegt, dass sich Schlegel bei zwei verschiedenen Anlässen mit der Bitte an Kopitar wandte, ihm ein slovenischdeutsches oder ein slovenisch-lateinisches Wörterbuch auszuleihen. Ein erstes Mal geschah es 1820 (Schlegel 1980: 300), ein zweites Mal ein Jahr danach (Schlegel 1980: 349). Der Lernprozess dürfte konkrete Formen angenommen haben, wenn sich Kopitar die Mühe nahm, seinen Landsmann und Sprachforscher aus Laibach, Franc Metelko (Brief vom 9. Januar 1821) zu bitten, ihm die Ergebnisse seiner Untersuchungen über die slovenische Orthographie zu schicken, weil er sie für Schlegel brauchte (Jagić 1897: 173).

Daraufhin revidierte Schlegel seine frühere These über die Verwandtschaft der europäischen Sprachen mit dem Sanskrit. In seinen Vorlesungen über Philosophie der Sprache und des Wortes, gehalten in Dresden im Dezember 1828, zählte er zu den mit dem Sanskrit verwandten Sprachen auch die slavischen:

Sollte ich nun etwa einen Versuch wenigstens andeuten, wie dieser Stufengang in seinen verschiedenen Epochen, in dem ganzen System und der unübersehlichen Menge aller über den Erdkreis verbreiteten Sprachen sich allenfalls in der Idee entwerfen oder nachweisen ließe; so würde ich dabei als von dem Sichersten und bekanntesten, zunächst von der ganzen indischen Sprach-Familie ausgehen, d. h. von allen jenen Sprachen, unter welchen die indische die erste Stelle einnimmt. Dahin gehört nebst der altpersischen, die gothischdeutsche, und die ihr zunächst verwandten skandinavischen Sprachen, dann die griechische und lateinische, nebst allen aus der letzten abgeleiteten, und endlich auch, nach dem Urtheil der sachkundigen Sprachforscher, die sämmtlichen slawischen Sprachen. (Schlegel 1846c: 70)

Schlegels Beschäftigung mit dem Slovenischen war forschungsbedingt und ist eigentlich durch Kopitars Initiative eingeleitet worden.

In der zehnten von seinen schon erwähnten Wiener Vorlesungen über die $G e-$ schichte der alten und neuen Literatur, überschrieben „Einige Worte über die Literatur der nördlichsten und östlichen Völker in Europa“, spricht Schlegel auch von den slavischen Sprachen und Nationen. Er stellt fest, dass die Slaven keine so reiche Poesie haben wie die germanischen und romanischen Völker und versucht sogar die Gründe dafür zu finden. Nach ihm liegt der Grund darin, dass die slavischen Völker an den Kreuzfahrten entweder nicht teilgenommen haben, oder nur in ganz geringem Ausmaß. Außerdem wagt Schlegel die Behauptung, die slavischen Sprachen ,scheinen auch von Natur weniger zur Poesie geneigt oder geeignet zu sein" (Schlegel 1846b: 31-37). Diese Vorlesung hat sich Kopitar mit Sicherheit angehört; das beweist sein oben zitierter Brief an Jakob Zupan vom 19. Februar 1812, verfasst bevor die Vorlesungen im Druck erschienen waren. Kopitar dürfte empfunden haben, dass Schlegel mit seiner Äußerung über die unpoetische Beschaffenheit der slavischen Sprachen die Grenzen der wissenschaftlichen Akzeptanz überschritten hatte. Im Gegenzug zu dieser von Arroganz gezeichneten Aussage verfasste er für das „Deutsche Museum“ zwei Aufsätze, die schon in den Überschriften die ironische Absicht 
ihres Autors ankündigen: Über die unmusikalische Beschaffenheit der deutschen Sprache $^{20}$ und Die deutsche Orthographie. Von einem Kosaken, an den Herausgeber. ${ }^{21}$ Fran Miklošič, der Kopitars kleinere Schriften herausgab, hat sich nicht getraut, den Titel des ersten Aufsatzes in der Originalfassung nachzudrucken und hat ihn grundlegend abgeändert: Aus der ,unmusikalischen Beschaffenheit der deutschen Sprache“ wurde die „musikalische Beschaffenheit der deutschen Sprache“ (Miklosich 1857: 178). Kopitar behauptet darin, dass die deutsche Sprache „unsingbar" sei, „weil sie ihre Worte meist auf Konsonanten endigt" und meint, dass in Westeuropa die italienische Sprache nach wie vor die Singsprache bleibt, während „in Osteuropa die slavische und die griechische es werden“222 (Miklosich 1857: 179).

Wie es schon beim ersten im „Deutschen Museum“ veröffentlichten Aufsatz Kopitars Über ein österreichisches Idiotikon der Fall war, so folgte auch bei diesem zweiten Aufsatz Über die unmusikalische Beschaffenheit der deutschen Sprache die Antwort des Herausgebers, in welcher Schlegel den Bemerkungen Kopitars „im ganzen durchaus nicht beistimmen kann“, obwohl dieser „im Einzelnen recht haben möge“" (Schlegel 1975a: 277).

Im Aufsatz Die deutsche Orthographie. Von einem Kosaken, an den Herausgeber wird von einem Kosaken erzählt, der sich nach St. Petersburg begibt, um dort von einem deutschen Lehrer Deutsch zu lernen. Die erste Frage, die der Kosak seinem Deutschlehrer stellte, war, ob die deutsche Sprache

[...] auch, wie die französische, von der alten und berühmten römischen abstamme, oder ob sie, wie die russische, eine barbarische, abkunftslose Sprache sei. (Miklosich 1857: 245-246)

Der Kosak musste noch dazu bald erfahren, das die deutsche Orthographie so „schlendrianisch“, „willkürlich“ und „,wetterwendisch“ ist, dass nicht nur die „Damen und unstudierte Leute“, sondern auch die „Gelehrten von Profession“ sich dabei schwer tun. Dagegen sei die russische Orthographie ganz einfach, wo eben ein Buchstabe einem Laut entspricht. So können die russischen Bauern, sobald sie das Alphabet kennen, sofort und für immer orthographisch richtig schreiben (Miklosich 1857: 246). Im übrigen Teil des Aufsatzes erteilt der Kosak den Deutschen Ratschläge für die Verbesserung ihrer Orthographie. Dieser Aufsatz war nicht nur der letzte Kopitars, sondern auch der allerletzte des „Deutschen Museums“, denn nach dem zwölften Heft des 2. Jahrgangs erschien die Zeitschrift nicht mehr. In der Anmerkung des Herausgebers, die unmittelbar auf Kopitars Aufsatz folgte, erklärt Schlegel, er werde an anderer Stelle zu diesem Aufsatz Stellung nehmen, da er in diesem Heft nicht mehr Raum finden konnte, was aber Absicht geblieben ist.

${ }^{20}$ Deutsches Museum 2/12 (1812), 533-535. Nachgedruckt in: Miklosich 1857: 178-180.

21 Deutsches Museum 4/12 (1813), 517-540. Nachgedruckt in: Miklosich 1857: 243-256.

22 Das Konzept von Kopitars Aufsatz Über die unmusikalische Beschaffenheit der deutschen Sprache wird in der Stadt- und Landesbibliothek Dortmund aufbewahrt. Daraus geht hervor, dass Kopitar den Artikel ursprünglich mit dem Sigel K gezeichnet hatte, dieses dann gestrichen und durch ein J [= Jernej] ersetzt hatte. S. dazu Anm. 34. 
Kopitar veröffentlichte im „Deutschen Museum“ außer den drei oben erwähnten Aufsätzen noch einen vierten: Kurze Nachricht über die Handschrift des altdeutschen Gedichtes Gottfried v. Bouillon, angeblich von Wolfram von Eschilbach (Bd. IV, 1813, 7. Heft, S. 517-540). Seine Mitarbeit beim „Deutschen Museum“ stellt ohne Zweifel, wie schon gesagt, das interessanteste und zugleich das merkwürdigste Kapitel der Kopitar-Schlegel Beziehung dar. Wenn man bedenkt, dass von der Zeitschrift nur zwei Jahrgänge erschienen sind, war die Mitarbeit Kopitars mit vier Aufsätzen allerdings beachtlich. In der Tat haben Schlegel-Forscher festgestellt, dass Kopitar zu den aktivsten Mitarbeitern des „Deutschen Museums“ gehörte (Schlegel 1975a: LXXIV). Es waren noch andere Beiträge vorgesehen, falls die Zeitschrift weiter erschienen wäre. Das geht aus einem Brief Kopitars an Dobrovský vom 14. Juni 1813 hervor, wo gesagt wird, dass Schlegel für sein Museum folgende Aufsätze wünscht: einen über die vorchristliche (= älteste) Staatsverfassung der Slaven, einen über die „wlachische Sprache“ und einen über das Albanische (Jagić 1885: 343-344).

In der Zeitspanne von 1820 bis 1823 gab Schlegel in Wien eine neue Zeitschrift heraus: die „Concordia“. Dass Kopitar an der neuen Zeitschrift nicht mitgearbeitet hat und diese von ihm in keinerlei Weise erwähnt wird, obwohl er zu dieser Zeit wie sein Briefwechsel beweist - mit Schlegel einen intensiven Kontakt hatte, ist mehr als verständlich, denn „Concordia“ war ein ausgesprochen katholisches Organ. Das geht aus der Vorrede des ersten Heftes deutlich hervor, wo gesagt wird, dass die Zeitschrift für das gebildete Publikum ,,in Österreich und in dem übrigen katholischen Deutschland“ bestimmt ist und dass sie „das ganze intellektuelle Leben der deutschen Nation [...] aus dem Standpunkte des Christentums betrachtet und bearbeitet" (Schlegel 1975a: 331-332). Noch dazu fand es Schlegel notwendig, in seinem einleitenden Beitrag Signatur des Zeitalters unter anderem die Herdersche Prophezeiung von der glorreichen Zukunft der Slaven in Abrede zu stellen (Schlegel 1967: 30).

In den 20er Jahren boten sich Schlegel gute Aussichten auf einen Posten an der Wiener Hofbibliothek. ${ }^{23}$ Am 21. August 1821 war nämlich der erste Kustos, Adam Ritter von Bartsch gestorben und Schlegel hoffte, dessen Stelle besetzen zu können. Das hätte bedeutet, dass Schlegel und Kopitar Arbeitskollegen geworden wären. Kopitar avancierte gerade am 21. August 1821 vom 1. Scriptor zum 4. Kustos. Schlegels Hoffnung zerschlug sich jedoch, als diese Kustosstelle aus Verwaltungsgründen Joh. Vesque von Püttlingen anvertraut wurde. Dieser war seit 1809 Kanzleidirektor

${ }^{23}$ Fr. v. Gentzs Brief an Pilat vom 21. August 1815: „Ich habe erfahren, daß der Custos der Wiener Bibliothek gestorben war, und macht dieserhalb einen Anwurf für Schlegel“" (Gentz 1868: 167). S. auch Fr. Schlegels Brief an Christine von Stransky vom 26. März 1824: „Es war mir seit lange Hoffnung zu der ersten Bibliothekarstelle hier gemacht worden [...]. Die Stelle war schon seit mehr als zwey Jahren unbesetzt, was mir aber eben nicht auffallend seyn konnte, da das oft hier geschiet [...]. Plötzlich aber erhält dieselbe ein Mann, an den niemand gedacht hat, und der auch gar nicht in diesem Fache bekannt ist, und der sie bloss bekommen hat, weil man ihn in einem anderen Büreau los seyn wollte." (Schlegel 1980: 653) 
beim Oberstkämmerer, dem Grafen Rudolf von Wrbna. Als der Graf am 30. Januar 1823 starb, beschloss man das Personal zu reduzieren und so wurde Vesque am 5. März 1824 zum Nachfolger Bartschs ernannt. ${ }^{24}$ Aber es dürften auch andere Gründe bestanden haben, weshalb Schlegel nicht für die Besetzung dieser vakanten Stelle berufen wurde: Sein Ungeschick und Misserfolg als Legationsrat bei der Österreichischen Gesandtschaft am Deutschen Bundestag in Frankfurt am Main in den Jahren 1815-1818 belasteten Schlegel nachhaltig. Man schätzte Schlegel als Denker und Schriftsteller nach wie vor, man wollte ihm aber offensichtlich keine Verantwortungsstelle im Staatsdienst anvertrauen. Ob Kopitar für diesen Posten lieber Schlegel gehabt hätte als Vesque, bleibt dahin gestellt. Darüber hat er sich in seiner Korrespondenz nie geäußert. Er hat Vesque von Püttlingen ein einziges Mal erwähnt, und zwar in einem Antwortschreiben an G. H. Pertz vom 29. Dezember 1827 - also fast vier Jahre nach dessen Ernennung - aber nur um seinem Korrespondenzpartner mitzuteilen, dass Bartsch verstorben war und dass ,ein Vesque von Püttlingen sein Nachfolger" geworden war. ${ }^{25}$

Im Kopitarschen literarischen Teilnachlass, der in der Österreichischen Nationalbibliothek in Wien aufbewahrt wird, befinden sich insgesamt sechs SchlegelBriefe an Kopitar (Sign.: Autogr. 12/71 und 140/24). Da die Briefpartner in derselben Stadt lebten und sich häufig trafen, bestehen die Briefe größtenteils aus kurzen Notizen. Sie umfassen den Zeitraum von 1815 bis 1827 und enthalten wertvolle Informationen über ihre Beziehungen. Die meisten Briefe (fünf) beziehen sich auf Kopitars Tätigkeit als Bibliothekar an der Hofbibliothek. Ein Brief enthält die Einladung an Kopitar, Schlegels Vorlesungen zu besuchen. In den Briefen wird Kopitar verschiedenartig angeredet: von „Hochgeehrter Herr" bis auf „Amice doctissime“. Es ist merkwürdig, dass Schlegel in einem Brief (Sommer 1820) Kopitar mit „Prof.“ betitelt. Vier Briefe (Sommer 1820, 20. Dezember 1820, Winter 1821, 17. April 1822) sind im 30. Band der Kritischen Friedrich-Schlegel Ausgabe ${ }^{26}$ veröffentlicht worden, während die übrigen zwei Schlegel-Briefe an Kopitar (19. Februar 1815, 2. April 1827), die noch unveröffentlicht geblieben sind, hier im Anhang ediert werden. Kopitar dürfte seinerseits keine Briefe an Schlegel adressiert haben; in seinem „Briefjournal“ finden sich dazu nämlich keine Belege (Glonar 1938).

In der Schlegeriana war Kopitar in drei verschiedenen Fällen genannt. Einmal als Mitarbeiter des „Deutschen Museums ${ }^{\text {‘27 }}$, ein zweites Mal als Fr. Schlegel-Korrespondent ${ }^{28}$ und ein drittes Mal im Zusammenhang mit einer anonymen Rezension

24 Der Vollständigkeit halber soll erwähnt werden, dass sich der in Brüssel am 12. November 1760 geborene Vesque von Püttlingen schon bei seiner Ankunft in Wien im Jahre 1804 um einen Posten an der Hofbibliothek beworben hatte. Er starb in Wien am 1. März 1829.

${ }^{25}$ Kopitar an G. H. Pertz, Wien, 29. Dezember 1827, in: Vasmer 1939: 92.

${ }^{26}$ Schlegel 1980: 300, 309, 349, 366.

27 Schlegel 1975a: LXXIV, LXXX-LXXXII, LXXXV, LXXXVII, 273-282, 374.

28 Schlegel 1980: 300, 309, 349, 366, 630, 634, 657, 669. 
des Werkes von Friedrich Wolf Horatius Erste Satyre, erschienen in der „Wiener allgemeinen Literaturzeitung“ (I, 1813, Nr. 60, S. 945-954), die Fr. Schlegel zugesprochen wurde. Der Grund für diese Zuordnung war ein Brief Friedrich Wolfs an Kopitar vom 23. November 1813, in dem es heißt:

Möchten Sie mir hierauf bald direkt mit der Post schreiben, zugleich (wo es etwa durch meinen edeln Freund Schlegel möglich ist) mir das Stück der Allg. Litt. Zeit. beizulegen, wo er mich über Horaz. I Satire noch etwas Neues ohne Zweifel gelehrt hat. Bloß durch Ihre Güte weiß ich davon. ${ }^{29}$

In diesem Zusammenhang behauptet der Germanist Ernst Behler, der verdienstvolle Herausgeber der Kritischen Friedrich-Schlegel-Ausgabe, in der Einleitung des 1. Bandes dieser Ausgabe, der Schlegels „Studien des klassischen Altertums“ gewidmet ist, dass eine sorgfältige Analyse des Stils und der Argumentationsweise ergibt, dass Schlegel nicht Verfasser dieser Rezension sein kann. Behler geht davon aus, dass Kopitars nicht erhaltene Nachricht an Wolf über Schlegels Autorschaft nur auf seiner Vermutung beruhen dürfte, nachdem damals die Meinung verbreitet war, Schlegel wäre ein aktiver Mitarbeiter der „Wiener allgemeinen Literaturzeitung“ gewesen. ${ }^{30}$ Es ist nicht meine Aufgabe, Behlers kritisch-philologische Deutungen zu kommentieren, ich möchte nur anmerken, dass Kopitar bei der „Wiener allgemeinen Literaturzeitung“, die gerade in diesem Jahr zu erscheinen begann, als Redakteur für Slavica und Graeca tätig war. ${ }^{31}$

Kopitar hatte auch nach dem Ableben Schlegels mit ihm zu tun. Schlegel starb am 11. Januar 1829. Drei Monate nach seinem Tod wandte sich der schon erwähnte Schlegel-Schützling Bucholtz im Namen der Witwe Schlegels, Dorothea, an Kopitar mit dem Anliegen, ob die Hofbibliothek bereit wäre, zwei Bände von Schlegels Handschriften anzukaufen. Kopitar antwortete auf das Angebot mit einem Brief vom 11. April 1829, in dem er feststellt, dass es sich dabei nur um bereits veröffentlichte Schriften handelte; für die Hofbibliothek hätten sie höchstens als Autographa Friedrich Schlegels einen Wert und mit Rücksicht darauf, könnte die Hofbibliothek wohl 10-12 Dukaten dafür bezahlen. ${ }^{32}$ Er fügte jedoch hinzu:

Wenn die resp[ektable] Witer es nicht vorzieht, sich durch ein Geschenk ein Denkmal in der Hofbib[liothek] zu stiften. ${ }^{33}$

Aus den herangezogenen Quellen tauchen deutliche Hinweise auf kritische Aspekte in der Kopitar-Schlegel Beziehung auf, wenigstens seitens Kopitars, dessen selbständige Persönlichkeit sich darin klar abzeichnet. Während Schlegel doch den „Gelehrten“ sah, den er zur Mitarbeit aufforderte und von dem er sich auch beein-

29 Schlegel 1979: CLXXXV-CLXXXVI.

30 Ebenda.

31 Vgl. Kopitar an Zupan, Wien, 14. Oktober 1812: „Dein Freund K. redigirt und sammelt Recens. für slavica et graeca, Hormayr et Schlegel Geschichte etc.“ (Jagić 1897: 247).

32 Kopitar an Bucholtz, Wien, 11. April 1829, in: Nordrhein-Westfälisches Staatsarchiv Münster, Nachlass Bucholtz, 247 (Kopitar).

${ }^{33}$ Ebenda. 
flussen lässt (Sanskrit, Muratori), äußert Kopitar eher Zweifel an der Wissenschaftlichkeit der Vorgehensweise Schlegels und fragt sich, ob er ein ,eigentlicher Gelehrter" sei, oder eher „ein Weltmann von gutem Kopf“.${ }^{34}$ Mit diesem Bedenken stand Kopitar nicht alleine da. So wertet zur gleichen Zeit Wilhelm von Humboldt die Vorlesungen Schlegels als „merkwürdige Erscheinungen am Wiener Horizont“" und sieht darin

[...] sophistische Rhetorik, die, von höchst einseitigem Gesichtspunkte aus, Philosophie und Kunst in eine bestimmte Form zu zwingen versuchte. (Schlegel 1975a: LXXIII)

Abschließend glaube ich sagen zu dürfen, dass die Überprüfung und die Neuauswertung der Quellen zur Kopitar-Schlegel Beziehung doch eine Notwendigkeit ist.

\title{
ANHANG
}

\section{Zwei ungedruckte Briefe Fr. Schlegels an B. Kopitar}

\author{
1. Fr. Schlegel an B. Kopitar, Wien, 19. Februar 1815 \\ Hochgeehrter Herr!
}

Wenn es möglich wäre, daß ich Champollions Pantheon Egyptien auf eine kurze Zeit ins Haus bekäme; so wäre mir dieses sehr erwünscht, und würde ich Ihnen auf das Dankbarste dafür verpflichtet seyn. - Haben Sie vielleicht schon nachgesehen, ob noch ein Zettel über ein fragment der Sapho in Ihrer Verwahrung ist, so bitte ich mich davon zu benachrichtigen. -

In der Hoffnung, Sie mit nächstem persönlich zu sehen, empfehle ich mich Ihrem freundschaftlichen Andenken; mit größter Hochachtung

Ihr ganz ergebenster

Wien, den 19ten Februar 1815

Fr. v. Schlegel

Dem Hochwohlgebornen

Herrn von Kopitar, Custos

der k.k. Bibliothek

${ }^{34}$ Kopitar an Dobrovský, Wien, 31. Januar 1813: „Quid tu ad Schlegelii responsum Jernejo datum im November seines Museums de geliebtes Kind musicaliori quam pulcherrimum idol mio! Equidem sub J. litera absconditus libera Kuhnii, germani slavisantis summi glottistae hic degentis verba audivi, Schlegelium ridentis ob tam germanas aures! Mit so einem haben wir ausgeredet, sagte er. Ego animavi, ut publice eadem fateatur. Warten Sie, es wird sich der J. schon rühren. Ego dixi me hoc vix sperare. Sie könnten Recht haben, denn er scheint kein eigentlicher Gelehrter, sondern ein Weltmann von gutem Kopf (nec hoc, nec illud). Aber einige Zeit müssen wir nur warten, ob er sich nicht meldet? Nemo me suspicatur! Ego sub cappa rideo." (Jagić 1885: 324) 
2. Fr. Schlegel an B. Kopitar, Wien, 2. April 1827

Amice Doctissime!

Durch beständiges Unwohlseyn $u$. viele Arbeit seit $8-14$ Tagen abgehalten, Ihnen diese Einladung selbst zu überbringen nehme ich mir die Freyheit, sie Ihnen zu übersenden; indem es mich sehr freuen würde, wenn Ihre Zeit Ihnen erlaubte meine Vorlesungen ein oder das andermal zu besuchen.

Vale et fave.

Ihr ergebenster Freund und

Diener Friedr. v. Schlegel

Wien

den 2 Apr. 1827.

Die Ankündigung im Inhaltsanzeiger hat vielleicht für einen oder den anderen immer auch ein Interesse und erlege ich daher einige Ex. desselben bey; auch für die Besucher der Bibliothek zum Verschicken.

\section{Literatur}

Bonazza 1980:

Bonazza 1983:

Bonazza 1988a:

Bonazza 1988b:

Bonazza 1990:

Ćurčin 1905:

Gentz 1868:

Glonar 1838:

Hafner 1957:

Hafner 1963:

Hafner 1969:
Sergio Bonazza, Bartholomäus Kopitar, Italien und der Vatikan (= Geschichte, Kultur und Geisteswelt der Slowenen XV), München Sergio Bonazza, Austro-Slavism as the Motive of Kopitar's Work, Slovene Studies 5, 155-164

Sergio Bonazza, Vuk Stef. Karadžić und der Austroslavismus, Europa Orientalis 7, 361-371 (Contributi italiani al X Congresso Internazionale degli slavisti, Sofia)

Sergio Bonazza, Neue Quellen zu Kopitars Wirken für Vuk Karadžić in Deutschland, Zeitschrift für Slawistik 33/5, 730-737

Sergio Bonazza, Kopitars Bedeutung für Kenntnis und Verbreitung der Kultur der Südslaven in Deutschland in der ersten Hälfte des 19. Jahrhunderts, Südosteuropa in der Wahrnehmung der deutschen Öffentlichkeit vom Wiener Kongreß (1815) bis zum Pariser Frieden (1856), hg. von Josip Matešić und Klaus Heitmann (= SüdosteuropaStudien 47), München, 165-172

Milan Curčin, Das serbische Volkslied in der deutschen Literatur, Leipzig

Briefe von Friedrich von Gentz an Pilat. Ein Beitrag zur Geschichte Deutschlands im XIX. Jahrhundert. Erster Band, hg. von Karl Mendelssohn-Bartholdy, Leipzig

Joža Glonar, Kopitarjev „Briefjournal“ 1816-1829, Glasnik Muzejskega društva za Slovenijo, 20/3-4, 142-150

Stanislaus Hafner, August Heinrich Hoffmann von Fallersleben und Bartholomäus Kopitar, Die Welt der Slaven 2, 183-200

Stanislaus Hafner, Das austro-slawische kulturpolitische Konzept in der ersten Hälfte des 19. Jahrhunderts, Österreichische Osthefte 5/6, 435-444

Stanislaus Hafner, Aus B. Kopitars Römischen Briefen an Josef Fesl, Studia Slovenica Monacensia. In honorem Antonii Slodnjak septuagenarii (= Geschichte, Kultur und Geisteswelt der Slowenen V), München, 29-42 
Hafner 1983:

Hafner 1997:

Jagić 1885:

Jagić 1897:

Kidrič 1939:

Kidrič 1941:

Mattson 1975:

Miklosich 1857:

Milović 1941a:

Milović 1941b:

Mittner 1964:

Mojašević 1990:

Pogačnik 1978:

Pogačnik 1982:

Schlegel 1812:

Schlegel 1846a:

Schlegel 1846b:

Schlegel 1846c:

Schlegel 1846d:

Schlegel 1966:

Schlegel 1967:

Schlegel 1975a:

Schlegel 1975b:

Schlegel 1979:
Stanislaus Hafner, Jernej Kopitar 1780-1844, Wegbereiter der deutsch-slawischen Wechselseitigkeit, hg. von Eduard Winter und Günther Jarosch, Berlin, 195-209

Stanislav Hafner, O Kopitarjevih poetoloških gledanjih, Zbornik ob sedemdesetletnici Franceta Bernika, Ljubljana, 177-185

Vatroslav Jagić, Briefwechsel zwischen Dobrowsky und Kopitar (1808-1828) (= Istočniki dlja istorii slavjanskoj filologii I) Berlin

Vatroslav Jagić, Neue Briefe von Dobrowsky, Kopitar und anderen Süd- und Westslaven (= Istočniki dlja istorii slavjanskoj filologii II), Berlin

France Kidrič, Zoisova korespondenca 1808-1809 (= Korespondence pomembnih Slovencev 1), Ljubljana

France Kidrič, Zoisova korespondenca 1809-1810 (= Korespondence pomembnih Slovencev 2), Ljubljana

Philip Mattson, Wilhelm von Humboldt und die Anfänge der Slavistik. Briefe an Kopitar, Zeitschrift für slavische Philologie 38, 303-323

Barth. Kopitars Kleinere Schriften, Erster Teil, hg. von Fr. Miklosich, Wien

Jevto M. Milović, Goethe, seine Zeitgenossen und die serbokroatische Volkspoesie (= Veröffentlichungen des Slavischen Instituts an der Friedrich-Wilhelms-Universität Berlin 30), Leipzig

Jevto M. Milović, Talvjs erste Übertragungen für Goethe und ihre Briefe an Kopitar (= Veröffentlichungen des Slavischen Instituts an der Friedrich-Wilhelms-Universität Berlin 33), Leipzig

Ladislao Mittner, Storia della letteratura tedesca. Dal Pietismo al Romanticismo (1700-1820), Torino

Miljan Mojašević, Jacob Grimm und die serbische Literatur und Kunst (= Marburger Studien zur Germanistik 14), Marburg

Jože Pogačnik, Bartholomäus Kopitar. Leben und Werk (= Geschichte, Kultur und Geisteswelt der Slowenen XV), München

Jože Pogačnik, Jernej Kopitar and the Issue of Austro-Slavism, Papers in Slavic Philology 2, 25-40

Deutsches Museum. Zweiter Jahrgang 1813, „Deutsches Museum“ 2. Bd., Elftes Heft, 463

Friedrich v. Schlegel, Sämmtliche Werke. Zweite Original-Ausgabe. Erster Band, Wien

Friedrich v. Schlegel, Sämmtliche Werke. Zweite Original-Ausgabe. Zweiter Band, Wien

Friedrich v. Schlegel, Sämmtliche Werke. Zweite Original-Ausgabe. Elfter Band, Wien

Friedrich v. Schlegel, Sämmtliche Werke. Zweite Original-Ausgabe. Fünfzehnter Band, Wien

Friedrich Schlegel, Studien zur Geschichte und Politik, hg. von Ernst Behler (= Kritische Friedrich-Schlegel-Ausgabe 7), München - Paderborn - Wien

Friedrich Schlegel, Concordia. Eine Zeitschrift. Hg. von Friedrich Schlegel. Mit einem Nachwort zur Neuausgabe von Ernst Behler, Darmstadt

Friedrich Schlegel, Charakteristiken und Kritiken II (1802-1829), hg. von Hans Eichner (= Kritische Friedrich-Schlegel-Ausgabe 3), München - Paderborn - Wien

Friedrich Schlegel, Studien zur Philosophie und Theologie, hg. von Ernst Behler und Ursula Struc-Oppenberg (= Kritische FriedrichSchlegel-Ausgabe 8), München - Paderborn - Wien

Friedrich Schlegel, Studien des klassischen Altertums, hg. von Ernst Behler (= Kritische Friedrich-Schlegel-Ausgabe 7), Paderborn - München - Wien 
Schlegel 1980: $\quad$ Friedrich Schlegel, Die Epoche der Zeitschrift Concordia (6. November 1818 - Mai 1823), hg. von Eugène Susini (= Kritische FriedrichSchlegel-Ausgabe 30), Paderborn - München - Wien

Schlegel 1995a: $\quad$ Fragmente zur Geschichte und Politik. Erster Teil, hg. von Ernst Behler (= Kritische Friedrich-Schlegel-Ausgabe 20), Paderborn - München - Wien

Schlegel 1995b: $\quad$ Fragmente zur Geschichte und Politik. Zweiter Teil, hg. von Ernst Behler (= Kritische Friedrich-Schlegel-Ausgabe 21), Paderborn München - Wien

Schulte-Kemminghausen 1958: Karl Schulte-Kemminghausen, Jacob Grimm und das serbo-

Vasmer 1938: $\quad$ Max Vasmer, Kopitars Briefwechsel mit Jakob Grimm (= Abhandlunkroatische Volkslied, Deutsches Jahrbuch für Volkskunde 4, 301-326 gen der Preußischen Akademie des Wissenschaften, Philosophisch-

Vasmer 1939: Historische Klasse, Jahrgang 1937, Nr. 7), Berlin Max Vasmer, Bausteine zur Geschichte der deutsch-slavischen geistigen Beziehungen I (= Abhandlungen der Preußischen Akademie der Wissenschaften, Philosophisch-historische Klasse, Jahrgang 1938, Nr.

Winter 1958: 6), Berlin

Winter 1968:

Eduard Winter, Eine grundlegende Urkunde des Austroslawismus, Zeitschrift für Slawistik 3, 107-124

$\begin{array}{ll}\text { Winter 1978: } & \text { Eduard Winter, Herder und die Entwicklung eines wirklichen Austro- } \\ \text { slavismus über Friedrich Schlegel, in Johann Gottfried Herder. Zur } \\ \text { Herder-Rezeption in Ost- und Südosteuropa, hg. von G. Ziegengeist, } \\ \text { H. Graßhoff und U. Lehmann (= Slawistische Studien und Texte), } \\ \text { Berlin, 205-211 }\end{array}$

Eduard Winter, Romantismus, Restauration und Frühliberalismus im österreichischen Vormärz, Wien

Ziegengeist 1983: G[erhard] Ziegengeist, Ein ungedruckter Brief B. Kopitars vom 17.X.1815 an J. S. Vater über Vuk Karadžić, Zeitschrift für Slawistik $28,522-532$

Ziegengeist 1988: G[erhard] Ziegengeist, Unbeachtete Zeugnisse zur frühen deutschen Karadžić-Rezeption, Zeitschrift für Slawistik 33, Nr. 5, 707-729

Sergio Bonazza

Nicolodistraße 7

I-39100 Bozen/Bolzano

sergio.bonazza@univr.it 
